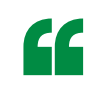

These results show a direct link between activity in faceselective parts of the fusiform gyrus and face perception g

\title{
PERCEPTION
}

\section{Facing reality}

In the human brain, parts of the fusiform gyrus respond strongly and selectively when a person sees a face, and damage to the fusiform gyrus can impair face perception or naming. However, it has been difficult to show that activity in these areas causes the perception of faces. In a new study, Parvizi et al. show that electrical perturbation of these areas causes a person to see faces as 'distorted', thereby demonstrating such a causal role.

The study involved a person in whom subdural electrodes had been implanted to localize the source of intractable seizures. The presence of the electrodes in the right inferior temporal lobe allowed the authors to use electrocorticography and electrical stimulation, combined with highresolution functional MRI (fMRI), to investigate the role of face-selective areas in the fusiform gyrus.

First, the authors used highresolution fMRI to map areas of the brain that responded to specific visual stimuli, and they located two distinct face-selective regions in the mid and posterior fusiform gyrus. Electrocorticographical recordings showed strong agreement with these findings, revealing that two electrodes $1 \mathrm{~cm}$ apart were placed over these two areas (called mFus-faces and pFus-faces). When the authors delivered electrical charge through this pair of electrodes, it produced a striking, face-specific effect; the subject reported that the faces of individuals in the room became 'distorted' or 'metamorphosed'.

Electrical perturbation of other electrodes or sham stimulation of these two electrodes did not cause similar distortion of faces, and perturbation of the mFus-faces and pFus-faces electrodes did not affect the appearance of objects other than faces. Surprisingly, it also did not impair the subject's ability to name famous faces seen on a computer screen, although the researchers did not specifically ask the subject to report distortions of these pictures.

These results show a direct link between activity in face-selective parts of the fusiform gyrus and face perception. The combination of approaches used here provides precise localization for recording and stimulation, and should help to answer further questions about cortical involvement in perception.

Rachel Jones

ORIGINAL RESEARCH PAPER Parvizi, J. et al. Electrical stimulation of human fusiform faceselective regions distorts face perception.

J. Neurosci. 32, 14915-14920 (2012)

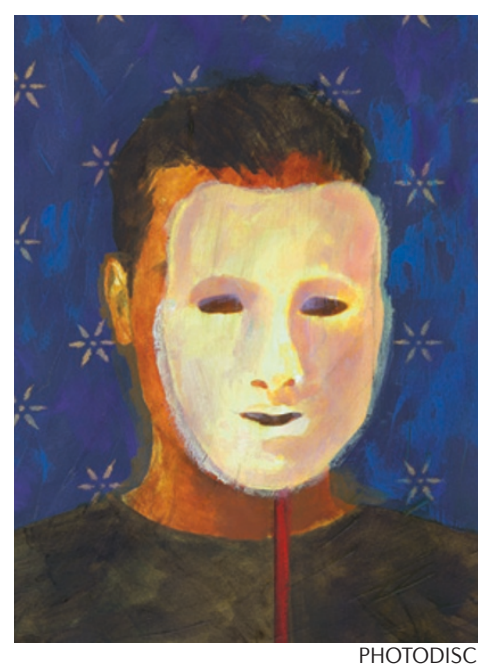

